\title{
Right Trigonal Cavernoma with Cysts and Superficial Siderosis
}

\author{
Dinesh Niti ${ }^{1} \quad$ Krishnan Nagarajan ${ }^{1, \odot}$ Mani Manoranjitha Kumari ${ }^{2}$ Lalith Kumar Balla \\ Sushila Chauhan ${ }^{2, \odot}$
}

${ }^{1}$ Department of Radiodiagnosis, Jawaharlal Institute of Postgraduate Medical Education \& Research (JIPMER), Puducherry, India

2Department of Neurosurgery, Jawaharlal Institute of Postgraduate Medical Education \& Research (JIPMER), Puducherry, India

\begin{abstract}
Address for correspondence Krishnan Nagarajan, MD, DM, Department of Radiodiagnosis, Jawaharlal Institute of Postgraduate Medical Education \& Research (JIPMER), Puducherry 605006, India (e-mail: Iknagarajan1@gmail.com).
\end{abstract}

\begin{abstract}
Keywords

- trigonal cavernomas

- intraventricular

masses

- superficial siderosis
\end{abstract}

\section{Case Summary}

A 26-year-old male patient presented with progressive holocranial headache, unresponsive to analgesics for 3 -month duration. The patient also complained of multiple episodes of projectile vomiting in the last 2 days. There was no history of fever, seizures, altered sensorium, or head trauma. Clinical examination revealed no focal neurologic deficits. Plain computed tomography axial sections showed a well-defined heterogenous hyperdense lesion measuring $\sim 2.5 \times 2.9 \times 3.5 \mathrm{~cm}$ (mediolateral $\times$ anteroposterior $\times$ craniocaudal) with peripheral calcific foci in the peritrigonal location of the right lateral ventricle. Vasogenic edema was seen involving the adjacent white matter with effacement of the cortical sulci. Perilesional fluid-density cysts were noted, the largest one extending medially and inferiorly into the basal cisterns. The cyst was seen compressing and displacing the midbrain anteriorly and to the left with aqueductal compression and upstream hydrocephalus. Ommaya shunt reservoir was placed the next day with a high cerebrospinal fluid opening pressure. Magnetic resonance imaging (MRI) of brain revealed a well-defined heterogenous right trigonal mass with extension into the right thalamus with features of moderate obstructive hydrocephalus (-Fig. 1). Susceptibilityweighted imaging revealed marked blooming within the lesion, lining the walls of the perilesional cysts and superficial siderosis (-Fig. 2). Intraoperatively, a gray thick-walled lesion with intralesional bleed (of varying ages), calcification, and immature vessels were noted in the right trigonal location extending into the thalamus and posterior limb of the internal capsule. Near-total excision of the lesion was done. Postoperative period was uneventful with no neurological deficits. The histopathology confirmed the lesion as cavernoma.

\section{Discussion}

Periventricular lesions in adults may present with symptoms of raised intracranial pressure (ICP) and may be due to lesions from the ependymal or subependymal origin published online April 19, 2021
DOI https://doi.org/

$10.1055 / \mathrm{s}-0041-1727424$ ISSN 2277-954X
(C) 2021. Neurological Surgeons' Society of India.

This is an open access article published by Thieme under the terms of the Creative Commons Attribution-NonDerivative-NonCommercial-License, permitting copying and reproduction so long as the original work is given appropriate credit. Contents may not be used for commercial purposes, or adapted, remixed, transformed or built upon. (https://creativecommons.org/licenses/by-nc-nd/4.0/).

Thieme Medical and Scientific Publishers Pvt. Ltd. A-12, 2nd Floor, Sector 2, Noida-201301 UP, India 


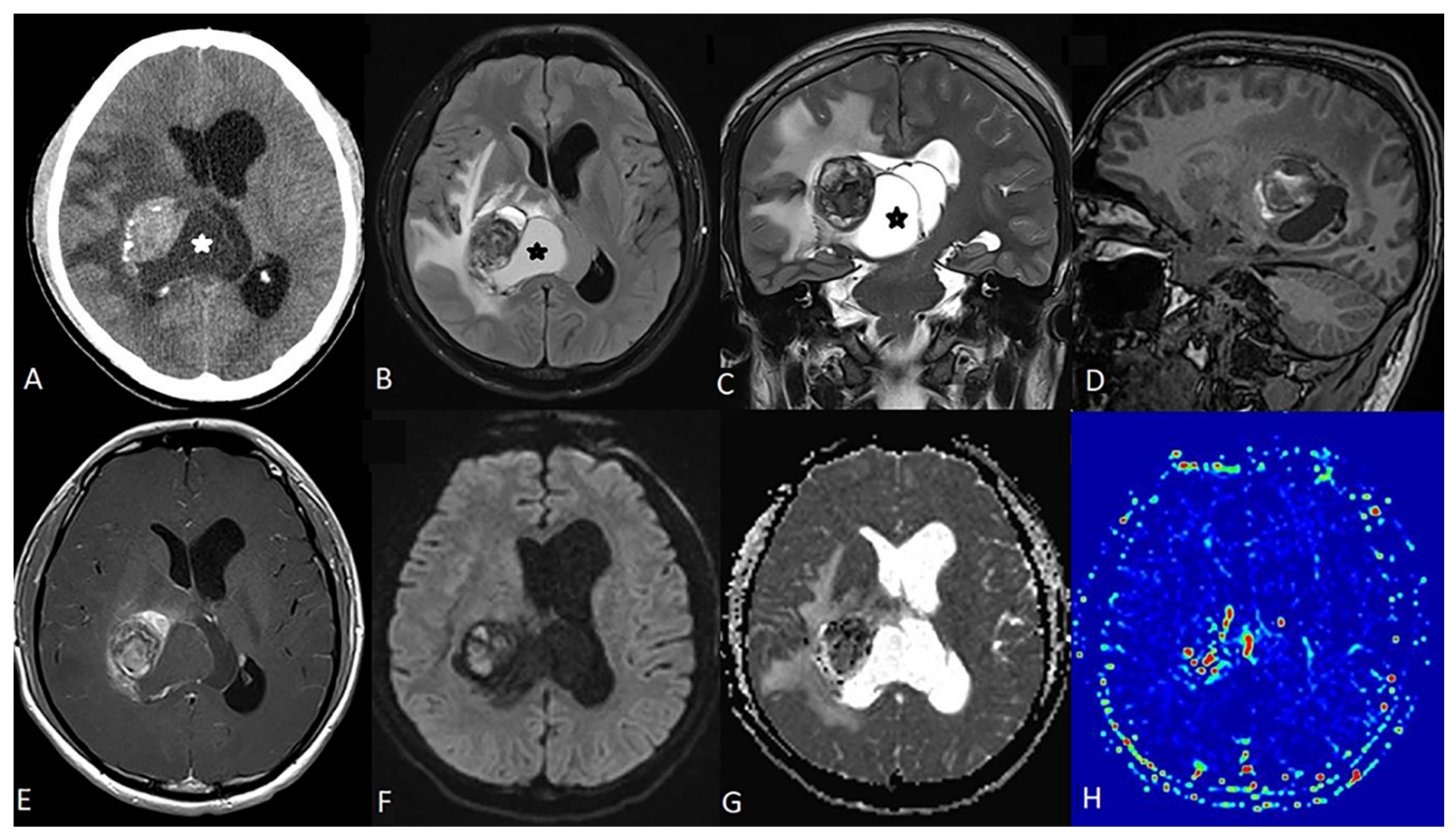

Fig. 1 Plain computed tomography axial (A), magnetic resonance imaging fluid-attenuated inversion recovery-FLAIR-axial (B), T2 coronal (C), and T1 sagittal (D) sections showing hyperdense lesion with calcific specks, T2 hypo- and T1 hyperintense areas, and medial cystic component $\left({ }^{*}\right)$. Postcontrast T1 axial (E) section showing partial peripheral enhancement. Diffusion-weighted imaging (F) and apparent diffusion coefficient $(\mathbf{G})$ showing heterogeneity and susceptibility artifacts with increased vascularity in perfusion (cerebral blood volume) map (H).

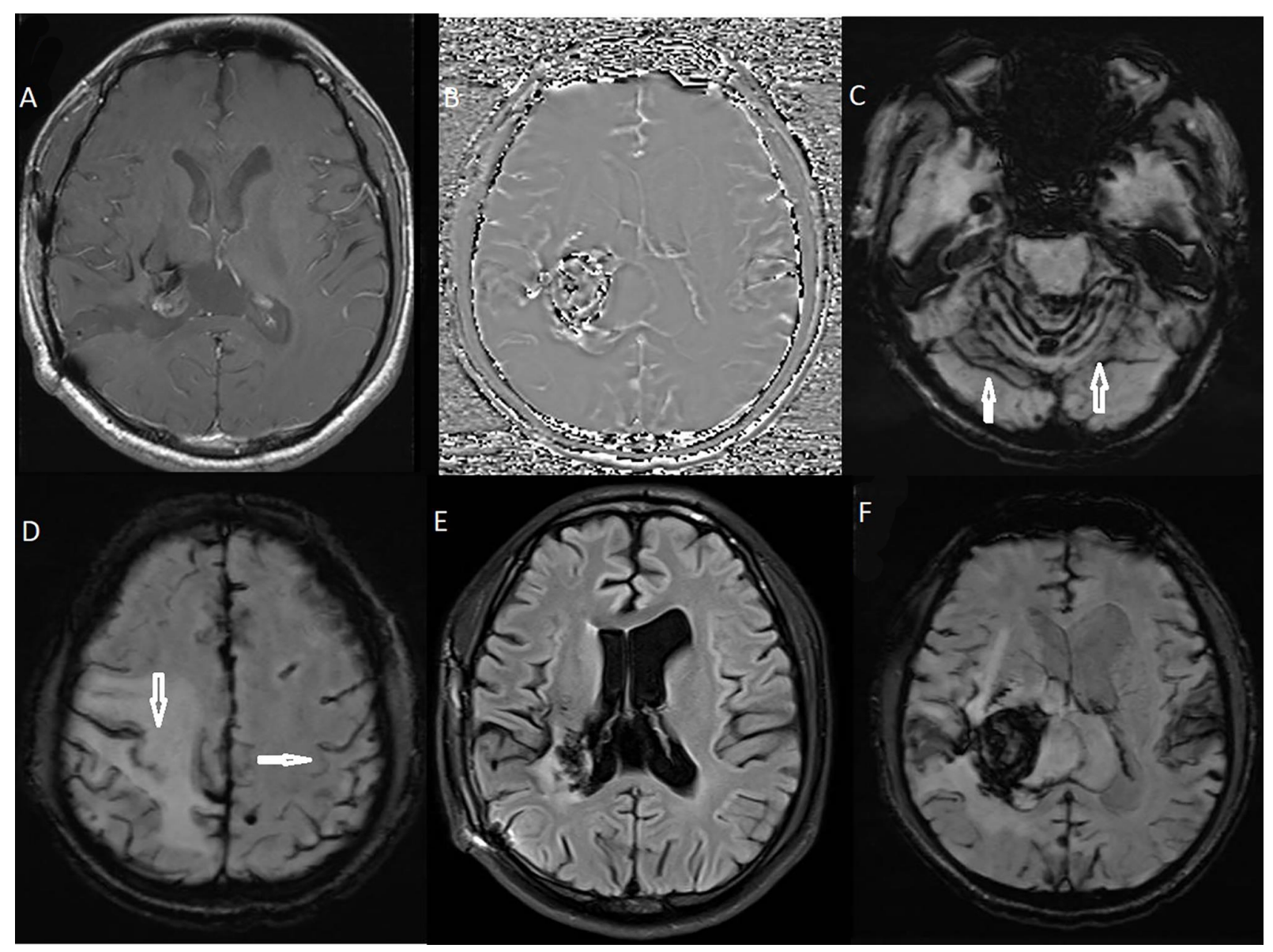

Fig. 2 Susceptibility-weighted imaging (SWI) axial (A) and SWI phase map (B) showing the blooming and hemorrhagic contents of the lesion. SWI images at cerebellar (C) and cortical (D) levels showing superficial siderosis along the cisterns, folia, and sulci (arrows). Postoperative fluid-attenuated inversion recovery-FLAIR (E) and postcontrast T1 (F) axial sections showing residual cavity and temporal lobe defect. 
including choroid plexus papilloma or carcinoma, meningiomas, ependymomas/subependymomas, and others like cavernomas. Peri- and intraventricular cavernomas have been reported to be 2.5 to $10.3 \%$ of brain cavernomas. ${ }^{1,2}$ Nearly a fifth (20\%) of them is located in the peritrigonal region. ${ }^{3}$ Recent reviews have shown that $\sim 20$ cases of trigonal cavernomas have been reported till now. ${ }^{1,4}$

Cavernomas are slow-flow vascular malformations and a type of hamartomas that are usually seen as intraparenchymal lesions, but rarely may arise on the meningeal or subependymal surface. The earliest report of intraventricular cavernomas was by Coin et al in $1977 .{ }^{5}$ Stavrinou et $\mathrm{al}^{6}$ in 2009 reviewed 13 cases of trigonal lesions reported till then out of 61 peri- and intraventricular cavernomas. The common presentations were raised ICP features, seizures, and bleeding. Sabat ${ }^{7}$ reported in 2010 the association of superficial siderosis in MRI of a case of intraventricular cavernoma.

Wu et $\mathrm{al}^{2}$ in 2018 summarized the cases of trigonal cavernomas reported in literature till then as less than 20. Wang et $\mathrm{al}^{4}$ in their report of 1,395 cases of intracranial and intraspinal cavernomas over a period of 14 years, found 12 cases of trigonal lesions, with 5 males and 12 females, average age of 33 years (7-53 years), and headache due to raised ICP as main symptom.

Shirvani and Hajimirzabeigi ${ }^{1}$ reviewed all intraventricular cavernomas reported and found 26 trigonal cavernomas out of 140 periventricular ones. Out of 26,13 were of right trigonal, 12 left side, and in one case, side was not mentioned. Mean age of presentation was 36.5 years with slight female predominance (69/61). Pediatric/adolescent patients formed $23 \%$ out of which $19 \%$ were in infants. Mean size of the lesions was not available in 45 patients and was $2.6 \mathrm{~cm}$ out of the rest 91 cases. Mass effect was seen in 91/131 (74\%) and intraventricular hemorrhage (IVH) in 30 (22.9\%). Out of 30 presented with IVH, 17 were in lateral ventricles, 7 in third, and 6 in fourth ventricles. Hydrocephalus was seen in $59(45 \%)$ cases out of which majority (31) were third ventricular lesions. Seizures were presenting symptoms in 16 cases $(16 / 131)$ in whom 13 were in lateral ventricle, and 8 in trigonal region.

Perilesional cysts are commonly seen in extra-axial lesions like meningiomas or schwannomas and occasionally in other lesions. The cystic component may increase the size and mass effect of the lesion and is usually decompressed along with the lesion. Sato and Kubota ${ }^{8}$ reported large cyst with cavernomas and hypothesized the cause to be recurrent hemorrhage and compared the cyst wall to the outer membrane noted in chronic subdural hematomas.

Superficial siderosis is caused by many lesions; the commonest causes are tumors, trauma, prior intracranial surgery, recurrent subarachnoid hemorrhage from aneurysms or vascular malformations, cerebral amyloid angiopathy (CAA), among others. ${ }^{9,10}$ Superficial siderosis is commonly cisternal but can be cortical as in CAA, or limited to only the supra- and/or infratentorial spaces. In Gradient $\mathrm{T}^{*}$ MRI or susceptibility-weighted MRI, they have characteristic appearance of "blooming" and are identified easily. Recurrent bleeds may be caused by many surface lesions but superficial siderosis is not a common feature of many intracranial neoplasms. Only chronic recurrent hemorrhages lead to superficial siderosis and hence the imaging findings of intraventricular mass with "blooming" of the lesion and the presence of superficial siderosis may help in characterization of the lesion as possible cavernoma.

\section{Conflict of Interest}

None declared.

\section{References}

1 Shirvani M, Hajimirzabeigi A. Intraventricular cavernous malformation: review of the literature and report of three cases with neuroendoscopic resection. J Neurol Surg A Cent Eur Neurosurg 2017;78(3):269-280

2 Wu J, Ren R, Qiu Z, Wang M. Multiple intracranial cavernous angiomas with a trigonal cavernous angioma mimicking glioma. J Craniofac Surg 2018;29(7):e635-e637

3 Ohbuchi H, Osaka Y, Ogawa T, et al. Trigonal cavernous malformation with intraventricular hemorrhage: a case report and literature review. J Med Invest 2012;59(3-4):275-279

4 Wang C, Zhao M, Deng X, Wang J, Jiang Z, Zhao J. Clinical features and neurosurgical treatment of trigonal cavernous malformations. Neurosurg Rev 2018;41(3):877-890

5 Coin CG, Coin JW, Glover MB. Vascular tumors of the choroid plexus: diagnosis by computed tomography. J Comput Assist Tomogr 1977;1(1):146-148

6 Stavrinou LC, Stranjalis G, Flaskas T, Sakas DE. Trigonal cavernous angioma: a short illustrated review. Acta Neurochir (Wien) 2009;151(11):1517-1520

7 Sabat SB. Intraventricular cavernous malformation with superficial siderosis. Arch Neurol 2010;67(5):638-639

8 Sato K, Kubota T. Large calcified cystic cavernous angioma in the thalamus-case report. Neurol Med Chir (Tokyo) 1995; 35(2):100-103

9 Fearnley JM, Stevens JM, Rudge P. Superficial siderosis of the central nervous system. Brain 1995;118(Pt 4):1051-1066

10 Kumar N. Superficial siderosis: associations and therapeutic implications. Arch Neurol 2007;64(4):491-496 